\title{
Floristic Survey of the Herbaceous-Shrub Layer of a Gallery Forest in Alto Paraíso de Goiás - Go, Brazil
}

\author{
Eduardo Chaves and Lucia Helena Soares-Silva* \\ ${ }^{1}$ Universidade de Brasília; Brasília - DF - Brasil. ${ }^{2}$ Departamento de Botânica; Universidade de Brasília; Brasília - \\ DF - Brasil
}

\begin{abstract}
Flowering species of the herbaceous and shrubby layer were sampled in a gallery forest at Portal da Chapada, Alto Paraíso de Goiás-GO in the Chapada dos Veadeiros during 12 months growing within 4.5 meters on either side of a raised pathway $2.4 \mathrm{Km}$ long. The collections were included to University of Brasilia Herbarium (UB). A total of 138 species (103 genera and 40 families) were recorded; the most species-rich families were Asteraceae (22), Poaceae (14), Rubiaceae (12), Fabaceae (11) and Melastomataceae (7). The richest family in number of genera was Asteraceae (19), followed by Poaceae (8), Fabaceae (8) and Rubiaceae (7). The results showed a meaningful increase in the Chapada dos Veadeiros flora, especially considering the herbaceous-shrubby layer of the gallery forests (223.6\%). Despite the richness of the flora, the number of endemics species in the Goiás State was only $1.5 \%$.
\end{abstract}

Key words: Floristic survey, Cerrado, Chapada dos Veadeiros

\section{INTRODUCTION}

The Cerrado biome of Central Brazil, extends across nine states (Bahia, Goiás, Maranhão, Mato Grosso, Mato Grosso do Sul, Minas Gerais, Piauí and Tocantins, as well in the, Federal District), including some disjunct areas in the states of Amapá, Amazonas, Pará, Paraná, Rondônia, Roraima and São Paulo (Cavalcanti 2002). This territory occupies an area of approximately two million square kilometers (Ribeiro and Walter 2001), an area far more extensive than, for example the Pantanal, and qualifies the Cerrado as the second largest Brazilian biome, being smaller only to the Amazon rainforest. Among the phytophysionomies present in this biome, the gallery forests, ecosystems associated with the water (Ribeiro and Walter 1998) have been object of various studies in recent decades, which is of fundamental importance to sustainable exploration and restoration of these environments.

Although the gallery and riverine forests occupy only $5 \%$ of the Cerrado biome, they contain nearly $33 \%$ of all known flowering plant species in the biome (Felfili et al. 2001), and although they are similar, these two forest types show some differences. These forest formations can be differentiated in three aspects (Ribeiro and Walter 2001): the width of the river which is adjacent to them, being narrower in the gallery forests, so that the tree crowns touch across the river (forming a gallery) while in the riparian forests, which can occur on the lake shores and the larger rivers (usually not exceeding 100 meters wide), the crowns do not touch, the deciduousness, which is much more evident in riparian forests and the floristic composition (with deciduous species predominant in the riparian forest and evergreen

\footnotetext{
* Author for correspondence: 1soares@unb.br
} 
species in the gallery forests). Gallery forests can also be divided into flooded and non-flooded (Ribeiro and Walter 1998).

In the gallery forests of the Federal District, the number of herbs and shrub species can be well represented compared to the floristic composition of the tree stratum. In a section of gallery forest which has a high degree of human disturbance along the Guará stream in the Guará Ecological Park-DF, 49 tree species and 56 shrub and herbaceous were found (Nogueira et al. 2002). In the Cafuringa Environmental Protection Area in the Northern Federal District, there exists at least 108 herb and shrub species and 125 trees in the gallery forests (Silva et al. 1996). Gallery forests of the Biosphere Nuclear Area Reserve in the Federal District have more than 700 terrestrial flowering plant species, with 374 herbaceous and shrubby species and 340 trees (Mendonça et al. 2000). Felfili et al. (2001) reported that the floral diversity in the gallery forests was, in general, very similar to an equivalent area in other tropical forests and richer than those of the adjacent savanna formations. Gallery forests also tend to have many endemic species (Silva Junior et al. 1998).

According to Felfili et al. (2001), the gallery forests of Cerrado biome have, in general, similar numbers of species in the herbaceous and shrub and tree layers. However, the studies involving the vegetation of these forests were almost exclusively in the tree stratum (Eira and Martins Netto 1998; Fonseca and Ribeiro 1998; Haridasan 1998; Souza 1999, Correia et al. 2001; Schiavini et al. 2001; Silva et al. 2001; Silva Júnior et al. 2001; Ribeiro and Walter 2001).

In the region of Alto Paraíso, various studies have contributed to improving the floral knowledge of the Chapada dos Veadeiros region, but they did not prioritize the herbaceous-shrub layer when undertaken in the gallery forests. Munhoz and Proença (1998) reported 1310 flowering plant species in various vegetation types in the region of Chapada dos Veadeiros; 55 of which were herbaceous and shrub species and 69 tree species in the gallery forests. Souza (1999) conducted a floristic survey on a farm in the municipality of Cavalcante-GO, adjacent to the Chapada dos Veadeiros National Park, sampling various vegetation types, and found five herbaceous, two shrub and 27 tree species for the gallery forests. Silva et al. (2001), identified in gallery forests in the region, 23 flowering plant species utilized by the local people, including six herbaceous and shrub species and 17 trees. In a floristic tree survey carried out in the Portal da Chapada, Alto Paraíso, Soares-Silva (2005) found 136 tree species in the same area of gallery forest of Couros river sampled in this present study, using the same collection criteria.

This work studied the herb-shrub species, along a section of gallery forest on the margins of the Couros river, and has been,, therefore a complementary study to that by Soares-Silva (2005) to provide more data on the same area, also allowing the comparison of data.

There is a clear discrepancy between the number of studies undertaken on tree species and the herbshrub layer, and consequently the herbs, shrubs and sub-shrubs are less well-known in the gallery forests. Knowledge on the floristic composition of the shrub layer may also be important in the projects of regenerating degraded areas, where not only trees but also shrub species can be used, information that corroborates that of Rezende (1998).

Herbaceous species may contribute to a rapid soil cover, improving the soil moisture conditions, increasing organic matter by accelerated decomposition, reducing erosion and also creating better conditions for development of tree species. In addition, the Chapada dos Veadeiros region has a well-developed ecotourism centre and for this activity, knowledge about the local flora would be of great importance, especially the herb-shrub layer of gallery forests, which are little known.

\section{MATERIAL AND METHODS}

\section{Area of study}

The study area is a forest formation with some flooded areas on the right bank of the Couros river, comprising 2.16 hectares, adjacent to an area of moist grassland. This area is located on the private property Portal da Chapada $\left(14^{\circ} 09\right.$ 'S, $47^{\circ}$ $35^{\prime} \mathrm{W}$, alt. $1164 \mathrm{~m}$ ) in the municipality of Alto Paraíso de Goiás-GO in the Chapada dos Veadeiros region, about $240 \mathrm{~km}$ from Brasília-DF (Fig. 1). The forest is intercut by small streams and is in an excellent condition of conservation, with well-developed specimens of copaiba (Copaifera langsdorfii Desf.), cajarana [Cabralea canjerana (Vell.) Mart.], peroba-branca (Aspidosperma spruceanum Benth. ex Muell. Arg.) and palmito (Euterpe edulis Mart.). 


\section{Floristic survey}

Part of the gallery forest was bisected by a trail, on which a wooden walkway (1m wide and $2.4 \mathrm{Km}$ long) was mounted, following the right bank of the Couros river. This catwalk, about $70 \mathrm{~cm}$ above the soil, was used as the reference point for the collections. Most of it passed througth the forest interior, and sometimes along the border (ecotone forest-wet grassland). Each day samples were collected, of all flowering plants in the herbaceous and shrub layer up to $4.5 \mathrm{~m}$ on each side of the walkway, throughout its extension. When the catwalk was along the forest border, the collections were made only of the forest side. This field procedure was repeated fortnightly for twelve months, totaling 25 sampling days.

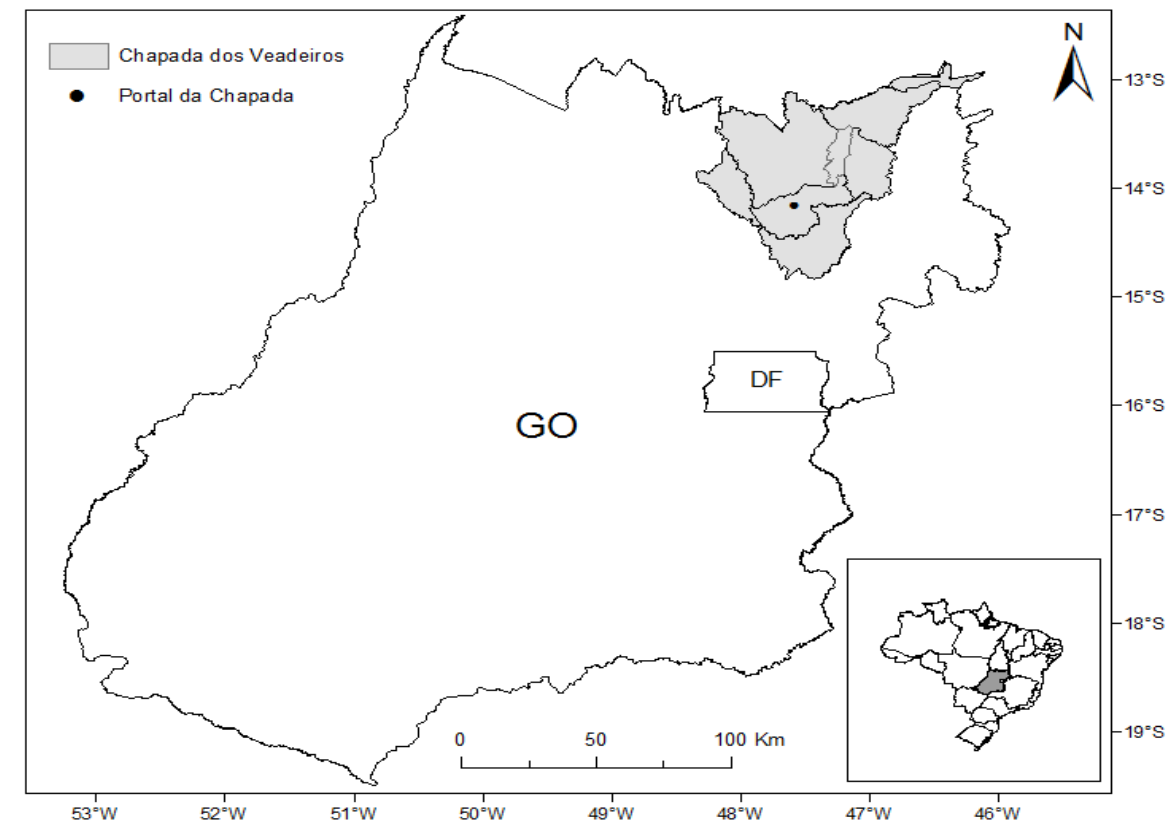

Figure 1 - Location of the State of Goiás, Chapada dos Veadeiros and Portal da Chapada in Brazil.

Species of terrestrial herbs, shrubs and semishrubs, flowering and /or fruiting, up to $2.5 \mathrm{~m}$ tall, had up to four samples collected, according to the availability of material. The most relevant characteristics of each species collected, such as height, colour and reproductive status were recorded in the field. Photographic records of specimens were taken with an Olympus digital camera with a resolution of 4 megapixels, to help the identification and create a photographic record.The specimens collected were processed according to standard methodology and were incorporated into the UB Herbarium (University of Brasilia).

The identifications were made by comparison with the herbarium material, consulting the relevant literature (Wanderley 1989; Filgueiras 1995, Judd et al. 1999; Barbosa and Amaral Jr. 2001, Cavalcanti et al. 2001; Barroso et al. 2002; Assis 2002; Carvalho-Silva and Cavalcanti 2002,
Lombardi 2002, Mendonca and Amaral Jr. 2002; Chautems 2003, Souza and Lorenzi 2005) and consultations with the specialists (Araceae, Asteraceae, Fabaceae, Lythraceae, Myrtaceae, Orchidaceae, Poaceae and Rubiaceae). The classification of the species was based on APG III (2009). The online databases consulted were Flora do Brasil, IPNI and MOBOT. In this study, the following definitions related to habit following Eiten (1992) were used:

\section{Herb}

The plant with herbaceous aerial shoots (devoid of a woody cylinder), which may be persistent (evergreen), pseudo-persistent, recurrent, biannual and annual.

\section{Shrub}

The plant with many fine aerial shoots (up to $3 \mathrm{~cm}$ in diameter), woody to the apex; subdivided into persistent, pseudo-persistent and recurrent. 


\section{Semi-shrub}

The plant with aerial stem and woody base and herbaceous in the distal part, subdivided into persistent, pseudo-persistent, base persistent, recurrent, biannual and annual.

\section{RESULTS AND DISCUSSION}

A total of 138 species were recorded belonging to
103 genera and 40 families (Table 1), a value similar to that found by Soares-Silva (2005) for the tree layer - 136 species in the same area. These results supported the study of Felfili et al (2001). The families with the highest number of species were Asteraceae (22 species), Poaceae (14), Rubiaceae (12), Fabaceae (11) and Melastomataceae (7), indicating that $47.8 \%$ of species were concentrated in $12.5 \%$ of the families.

Table 1 - Herbaceous and shrub flowering plants of the gallery forest on the right side of the Couros river, at the Portal da Chapada, in Alto Paraíso de Goiás-GO. UB - University of Brasília Herbarium. LF - life form: Ss - Semishrub, Sh - Shrub, He - Herb. Habitat: GF - Gallery Forest Interior, FB- Gallery Forest Border; Fl - Flower; Fr Fruit. Numbers $1-12=$ months of the year.

\begin{tabular}{|c|c|c|c|c|c|}
\hline Family/Species & UB & LF & Habitat & Fl. & Fr. \\
\hline \multicolumn{6}{|l|}{ Acanthaceae } \\
\hline Aphelandra longiflora (Lindl.) Profice & 16382 & Ss & GF & $4-6$ & $7-10$ \\
\hline Justicia chrysotrichoma Pohl ex Nees & 19598 & Ss & $\mathrm{FB}^{*}$ & $6-8$ & - \\
\hline Justicia irwinii Wassh. & 16371 & Ss & GF & $4-7$ & $7-8$ \\
\hline Justicia lanstyakii Rizzini & 19491 & Ss & $\mathrm{FB}^{*}$ & $6-9$ & 9 \\
\hline Lepidagathis floribunda (Pohl) Kameyama & 16375 & Ss & GF & $3-7$ & $7-9$ \\
\hline \multicolumn{6}{|l|}{ Alstroemeriaceae } \\
\hline Alstroemeria stenopetala Schenk & 19403 & $\mathrm{He}$ & FB & $2-4$ & $4-6$ \\
\hline \multicolumn{6}{|l|}{ Amaranthaceae } \\
\hline Gomphrena sp. & 19463 & $\mathrm{He}$ & FB & 6 & - \\
\hline \multicolumn{6}{|l|}{ Apocynaceae } \\
\hline Mandevilla emarginata (Vell.) C. Ezcurra & 19386 & Ss & $\mathrm{FB}^{*}$ & $2-7$ & $7-8$ \\
\hline \multicolumn{6}{|l|}{ Araceae } \\
\hline Philodendron mayoi E. G. Gonç. & 19521 & $\mathrm{He}$ & GF & $9-10$ & 11 \\
\hline Philodendron wullschlaegelii Schott & 19522 & $\mathrm{He}$ & GF & $2-3$ & 8 \\
\hline \multicolumn{6}{|l|}{ Araliaceae } \\
\hline Hydrocotyle quinqueloba Ruiz and Pav. & 16389 & $\mathrm{He}$ & GF, FB & $7-5$ & $3-9$ \\
\hline \multicolumn{6}{|l|}{ Asteraceae } \\
\hline Ageratum fastigiatum (Gardner) R. M. King and H. Rob. & 19482 & $\mathrm{He}$ & $\mathrm{FB}^{*}$ & $6-8$ & 7 \\
\hline Aspilia foliacea (Spreng.) Baker & 19266 & $\mathrm{He}$ & $\mathrm{FB}^{*}$ & 12 & - \\
\hline $\begin{array}{l}\text { Autroeupatorium inulaefolium (Kunth) R. M. King and } \\
\text { H. Rob. }\end{array}$ & 17707 & Ss & FB & $4-5$ & - \\
\hline Calea teucriifolia (Gardner) Baker & 19358 & Ss & FB* & $1-8$ & - \\
\hline $\begin{array}{l}\text { Campuloclinium megacephalum (Mart. ex Baker) R. M. } \\
\text { King and H. Rob. }\end{array}$ & 19381 & $\mathrm{He}$ & $\mathrm{FB}^{*}$ & 2 & - \\
\hline Chresta angustifolia Gardner & 17877 & $\mathrm{He}$ & GF & 10 & - \\
\hline $\begin{array}{l}\text { Chromolaena chaseae (B. L. Rob.) R. M. King and H. } \\
\text { Rob. }\end{array}$ & 19426 & Ss & $\mathrm{FB}^{*}$ & $4-6$ & $6-8$ \\
\hline $\begin{array}{l}\text { Chromolaena stachyophylla (Spreng.) R. M. King and H. } \\
\text { Rob. }\end{array}$ & 19284 & Ss & $\mathrm{FB}^{*}$ & 11 & 12 \\
\hline Chrysolaena desertorum (Mart. ex DC.) Dematt. & 19251 & Ss & $\mathrm{FB}^{*}$ & 10 & - \\
\hline Elephantopus mollis Kunth & 19462 & $\mathrm{He}$ & $\mathrm{FB}^{*}$ & $5-6$ & - \\
\hline Grazielia intermedia (DC.) R. M. King and H. Rob. & 19360 & Ss & FB & $1-2$ & - \\
\hline
\end{tabular}


(Cont. Table 1)

\begin{tabular}{|c|c|c|c|c|c|}
\hline Family/Species & UB & $\mathbf{L F}$ & Habitat & Fl. & Fr. \\
\hline Lepidaploa aurea (Mart. ex DC.) H. Rob. & 16372 & Ss & FB & $4-6$ & $5-8$ \\
\hline Lessingianthus argyrophyllus (Less.) H. Rob. & 19370 & Ss & $\mathrm{FB} *$ & 1 & - \\
\hline Lessingianthus ligulifolius (Mart. ex DC.) H. Rob. & 19283 & Ss & $\mathrm{FB}^{*}$ & 2 & - \\
\hline $\begin{array}{l}\text { Raulinoreitzia tremula (Hook and Arn.) R. M. King and } \\
\text { H. Rob. }\end{array}$ & 19483 & Ss & $\mathrm{FB} *$ & 6 & - \\
\hline Senecio adamantinus Bong. & 19301 & $\mathrm{He}$ & $\mathrm{FB} *$ & 12 & - \\
\hline Stevia heptachaeta DC. & 19461 & $\mathrm{He}$ & $\mathrm{FB} *$ & 5 & - \\
\hline Symphyopappus reticulatus Baker & 19351 & Ss & $\mathrm{FB}^{*}$ & 1 & - \\
\hline Vernonanthura ferruginea (Less.) H. Rob. & 19511 & Ss & GF & $5-9$ & $7-8$ \\
\hline Viguiera grandiflora (Gardner) Gardner & 19250 & Ss & $\mathrm{FB} *$ & 10 & - \\
\hline \multicolumn{6}{|l|}{ Bromeliaceae } \\
\hline Aechmea bromeliifolia (Rudge) Baker & 19277 & $\mathrm{He}$ & GF & $5-10$ & $11-12$ \\
\hline Bromelia antiacantha Bertol. & 19520 & $\mathrm{He}$ & GF & 12 & 12 \\
\hline \multicolumn{6}{|l|}{ Burmanniaceae } \\
\hline Apteria aphylla (Nutt.) Barnhart ex Small & 19503 & $\mathrm{He}$ & GF & 7 & - \\
\hline Dictyostega orobanchoides (Hook.) Miers & 19435 & $\mathrm{He}$ & GF & $4-5$ & - \\
\hline \multicolumn{6}{|l|}{ Campanulaceae } \\
\hline Centropogon cornutus (L.) Druce & 17723 & Ss & GF & $6-2$ & - \\
\hline Siphocampylus corymbiferus Pohl & 19492 & Ss & GF & $6-7$ & - \\
\hline \multicolumn{6}{|l|}{ Chloranthaceae } \\
\hline Hedyosmum brasiliense Miq. & 16378 & Ss & $G F$ & - & $4-5$ \\
\hline \multicolumn{6}{|l|}{ Clusiaceae } \\
\hline Kielmeyera pumila Pohl & 19336 & Ss & $\mathrm{FB} *$ & 12 & - \\
\hline \multicolumn{6}{|l|}{ Commelinaceae } \\
\hline Commelina erecta $\mathrm{L}$. & 19319 & $\mathrm{He}$ & FB & $1-4$ & 4 \\
\hline Commelina obliqua Vahl & 16381 & $\mathrm{He}$ & GF & 4-6 10-11 & $4-5$ \\
\hline \multicolumn{6}{|l|}{ Cyperaceae } \\
\hline Cyperus aggregatus (Willd.) Endl. & 17734 & $\mathrm{He}$ & $\mathrm{GF}$ & $4-11$ & - \\
\hline Rhynchospora armerioides J. Presl and C. Presl & 19278 & $\mathrm{He}$ & $\mathrm{FB} *$ & 11 & - \\
\hline Rhynchospora brasiliensis Boeckeler & 19399 & $\mathrm{He}$ & FB & $9-5$ & $8-5$ \\
\hline Rhynchospora exaltata Kunth & 17735 & $\mathrm{He}$ & GF, FB & $1-12$ & $1-12$ \\
\hline Scleria latifolia $\mathrm{Sw}$. & 16383 & $\mathrm{He}^{* *}$ & GF & $1-12$ & $1-12$ \\
\hline \multicolumn{6}{|l|}{ Eriocaulaceae } \\
\hline Paepalanthus amoenus (Bong.) Körn. & 19398 & $\mathrm{He}$ & $\mathrm{FB} *$ & $2-6$ & $6-7$ \\
\hline Paepalanthus chlorocephalus Silveira & 19480 & $\mathrm{He}$ & $\mathrm{FB} *$ & 6 & - \\
\hline Syngonanthus caulescens (Poir.) Ruhland & 17733 & $\mathrm{He}$ & GF & 10 & - \\
\hline \multicolumn{6}{|l|}{ Erythroxylaceae } \\
\hline Erythroxylum deciduum A. St.-Hil. & 19337 & Sh & FB & $8-1$ & $9-2$ \\
\hline Erythroxylum suberosum A. St.- Hil. & 17873 & $\mathrm{Sh}$ & $\mathrm{FB} *$ & $8-12$ & $9-1$ \\
\hline \multicolumn{6}{|l|}{ Euphorbiaceae } \\
\hline Croton antisyphiliticus Mart. & 19248 & Ss & $\mathrm{FB} *$ & $10-11$ & $11-12$ \\
\hline Sapium glandulosum (L.) Morong & 19268 & $\mathrm{Sh}$ & FB & $10-11$ & 12 \\
\hline Microstachys bidentata (Mart. and Zucc.) Esser & 19354 & $\mathrm{He}$ & $\mathrm{FB} *$ & 1 & 5 \\
\hline Sebastiania myrtilloides (Mart.) Pax & 19357 & $\mathrm{He}$ & $\mathrm{FB}^{*}$ & 1 & 1 \\
\hline \multicolumn{6}{|l|}{ Fabaceae } \\
\hline Aeschynomene paniculata Willd. ex Vogel & 16373 & $\mathrm{He}$ & $\mathrm{FB} *$ & $4-5$ & $4-5$ \\
\hline Bionia coriacea (Nees and Mart.) Benth. & 17731 & Ss & FB & $3-9$ & $5-6$ \\
\hline Chamaecrista desvauxii (Collad.) Killip & 19400 & Ss & $\mathrm{FB} *$ & $5-6,10-2$ & 6,11 \\
\hline
\end{tabular}


(Cont. Table 1)

\begin{tabular}{|c|c|c|c|c|c|}
\hline FAMILY/SPECIES & UB & LF & Habitat & Fl. & Fr. \\
\hline Chamaecrista nictitans (L.) Moench & 19428 & Ss & $\mathrm{FB}^{*}$ & 3 & 3 \\
\hline $\begin{array}{l}\text { Chamaecrista paniculata (Benth.) H. S. Irwin and } \\
\text { Barneby }\end{array}$ & 19519 & Ss & GF & $5-7$ & 1 \\
\hline Crotalaria micans Link & 19429 & Ss & FB & 3 & - \\
\hline Crotalaria velutina Benth. & 19382 & Ss & $\mathrm{FB}^{*}$ & 2 & - \\
\hline Eriosema brevipes Grear & 19340 & Ss & $\mathrm{FB}^{*}$ & $12-3$ & $12-3$ \\
\hline Periandra mediterranea (Vell.) Taub. & 17729 & Sh & GF & $4-10$ & $5,8-12$ \\
\hline Senna corifolia (Benth.) H. S. Irwin and Barneby & 16386 & $\mathrm{Sh}$ & $\mathrm{FB}^{*}$ & $4-5$ & 5 \\
\hline Stylosanthes guianensis (Aubl.) Sw. & 17710 & $\mathrm{He}$ & $\mathrm{FB}^{*}$ & $1-5$ & - \\
\hline $\begin{array}{l}\text { Gesneriaceae } \\
\text { Sinningia elatior (Kunth) Chautems }\end{array}$ & 16384 & $\mathrm{He}$ & $\mathrm{GF} \cdot \mathrm{FB}$ & $3-5$ & _ \\
\hline Iridaceae & & & & & \\
\hline Bermudiana incurvata (Gardn.) Kuntze & 19365 & $\mathrm{He}$ & $\mathrm{FB}^{*}$ & $12-1$ & $12-1$ \\
\hline Lamiaceae & & & & & \\
\hline Amasonia campestris (Aubl.) Moldenke & 19369 & Ss & $\mathrm{FB} *$ & $1-4$ & - \\
\hline Hypenia calycina (Pohl ex Benth.) Harley & 17730 & Ss & FB & $4-10$ & $6-7$ \\
\hline Hypenia sp. & 19465 & Ss & FB & 5 & 5 \\
\hline Hyptis cuneata Pohl ex Benth. & 17736 & $\mathrm{He}$ & $\mathrm{FB}^{*}$ & 10 & - \\
\hline Hyptis rubiginosa Mart. ex Benth. & 19474 & Ss & $\mathrm{FB}^{*}$ & 5 & - \\
\hline Marsypianthes montana Benth. & 19388 & Ss & $\mathrm{FB}^{*}$ & $2-7$ & $7-8$ \\
\hline $\begin{array}{l}\text { Lentibulariaceae } \\
\text { Utricularia hispida Lam. }\end{array}$ & 19376 & $\mathrm{He}$ & $\mathrm{FB} *$ & 2 & - \\
\hline Lythraceae & & & & & \\
\hline Cuphea spermacoce A. St.-Hil. & 19285 & Ss & $\mathrm{FB}^{*}$ & $10-3$ & $10-3$ \\
\hline Diplusodon incanus Gardn. & 19443 & Ss & $\mathrm{FB}^{*}$ & 4 & - \\
\hline $\begin{array}{l}\text { Malpighiaceae } \\
\text { Tetrapterys mucronata } \text { Cav. }\end{array}$ & 17727 & Ss & FB & $9-10$ & - \\
\hline $\begin{array}{l}\text { Malvaceae } \\
\text { Byttneria jaculifolia Pohl } \\
\text { Pavonia malacophylla (Link and Otto) Garcke } \\
\text { Sida glomerata Cav. }\end{array}$ & $\begin{array}{l}19343 \\
17719 \\
19437\end{array}$ & $\begin{array}{l}\text { Ss } \\
\text { Ss } \\
\text { Ss }\end{array}$ & $\begin{array}{l}\text { FB* } \\
\text { GF } \\
\text { GF }\end{array}$ & $\begin{array}{l}12 \\
5 \\
4\end{array}$ & $\begin{array}{l}- \\
9 \\
-\end{array}$ \\
\hline Melastomataceae & & & & & \\
\hline Leandra lacunosa Cogn & 19246 & $\mathrm{Sh}$ & FB & $10-11$ & 11 \\
\hline Leandra polystachya (Naudin.) Cogn & 17878 & $\mathrm{Sh}^{* *}$ & FB & $10-11$ & $10-11$ \\
\hline Leandra salicina (DC.) Cogn & 19419 & $\mathrm{Sh}$ & GF & - & 3 \\
\hline Miconia albicans (Sw.) Triana & 17870 & $\mathrm{Sh}$ & FB & $10-11$ & - \\
\hline Miconia chamissois Naud. & 19245 & Ss & $\mathrm{FB}$ & - & $10-11$ \\
\hline Microlicia euphorbioides Mart. & 19349 & Sh & FB & 1 & - \\
\hline Tibouchina aegopogon (Naudin) Cogn & 19334 & Ss & $\mathrm{FB}^{*}$ & 12 & - \\
\hline $\begin{array}{l}\text { Myrsinaceae } \\
\text { Cybianthus goyazensis Mez }\end{array}$ & 19411 & Ss & GF & 9 & $9-5$ \\
\hline Myrtaceae & & & & & \\
\hline Campomanesia eugenioides (Cambess.) D. Legrand & 19252 & Ss & GF & $10-12$ & 12 \\
\hline Campomanesia pubescens (mart. ex DC.) O. Berg & 19253 & Ss & FB & $9-10$ & 10 \\
\hline Myrcia tocantinensis Glaziou (nom. nud.) & 19341 & Ss & FB & 12 & 12 \\
\hline Orchidaceae & & & & & \\
\hline Epidendrum secundum Jacq. & 19422 & $\mathrm{He}$ & FB & $3-8$ & $6-8$ \\
\hline Malaxis cf. excavata (Lindl.) Kuntze & 19433 & $\mathrm{He}$ & GF & - & 4 \\
\hline Oeceoclades maculata (Lindl.) Lindl. & 19504 & $\mathrm{He}$ & GF & - & $7-8$ \\
\hline Prescottia stachyodes (Sw.) Lindl. & 19502 & $\mathrm{He}$ & GF & $7-8$ & 9 \\
\hline
\end{tabular}


(Cont. Table 1)

\begin{tabular}{|c|c|c|c|c|c|}
\hline FAMILY/SPECIES & UB & LF & Habitat & Fl. & Fr. \\
\hline \multicolumn{6}{|l|}{ Oxalidaceae } \\
\hline Oxalis diamantinae $\mathrm{R}$. Knuth & 19265 & $\mathrm{He}$ & FB & 11 & - \\
\hline \multicolumn{6}{|l|}{ Piperaceae } \\
\hline Piper aduncum L. & 19481 & Sh & GF & $6-9$ & 9 \\
\hline Piper cernuит Vell. & 19348 & Sh & GF & $1,7-9$ & - \\
\hline Piper crassinervium Kunth & 19505 & $\mathrm{Sh}$ & GF & $7-8$ & $7-8$ \\
\hline Piper xylosteoides (Kunth) Steud. & 16377 & $\mathrm{Sh}$ & GF & $4-11$ & $8-12$ \\
\hline \multicolumn{6}{|l|}{ Poaceae } \\
\hline Echinolaena inflexa (Poir.) Chase & 19430 & $\mathrm{He}$ & $\mathrm{FB}^{*}$ & $3-5$ & $5-6$ \\
\hline Ichnanthus bambusiflorus (Trin.) Döll & 19309 & $\mathrm{He}$ & FB & $12-1$ & - \\
\hline Ichnanthus aff. longiglumis $\mathrm{Mez}$ & 19280 & $\mathrm{He}$ & $\mathrm{FB} *$ & $12-3$ & - \\
\hline Ichnanthus mollis Ekman & 19362 & $\mathrm{He}$ & $\mathrm{FB}, \mathrm{GF}$ & $1-7$ & - \\
\hline Ichnanthus sp. & 17720 & $\mathrm{He}$ & GF & 5 & - \\
\hline Loudetiopsis chrysothrix (Nees) Conert & 19431 & $\mathrm{He}$ & $\mathrm{FB} *$ & $3-6$ & - \\
\hline Ocellochloa rudis (Nees) Zuloaga and Morrone & 19361 & $\mathrm{He}$ & $\mathrm{FB}, \mathrm{GF}$ & $1-5$ & - \\
\hline Oplismenus hirtellus (L.) P. Beauv. & 16380 & $\mathrm{He}$ & GF & $4-5$ & - \\
\hline Panicum parvifolium Lam. & 17732 & $\mathrm{He}$ & GF & 10 & - \\
\hline Panicum pilosum $\mathrm{Sw}$. & 19449 & $\mathrm{He}$ & GF & 4 & - \\
\hline Panicum sp. & 19397 & $\mathrm{He}$ & $\mathrm{FB}, \mathrm{GF}$ & $1-3,8$ & - \\
\hline Paspalum coryphaeum Trin. & 19372 & $\mathrm{He}$ & FB & 1 & - \\
\hline Paspalum sp. & 19487 & $\mathrm{He}$ & FB & 6 & - \\
\hline Trachypogon spicatus (L. f.) Kuntze & 19439 & $\mathrm{He}$ & $\mathrm{FB}^{*}$ & 4 & - \\
\hline \multicolumn{6}{|l|}{ Polygalaceae } \\
\hline Polygala poaya Mart. & 17871 & Ss & GF, FB & $6-10$ & - \\
\hline \multicolumn{6}{|l|}{ Rubiaceae } \\
\hline Cordiera concolor (Cham.) Kuntze & 17726 & $\mathrm{Sh}$ & GF & $4,8-11$ & $6-12$ \\
\hline Cordiera sessilis (Vell.) Kuntze & 17879 & Sh & GF & $10-11$ & - \\
\hline Borreria latifolia (Aubl.) K. Schum. & 19332 & $\mathrm{He}$ & $\mathrm{GF}, \mathrm{FB}$ & $12-6$ & $3-6$ \\
\hline Coccocypselum aureum (Spreng.) Cham. and Schltdl. & 19264 & $\mathrm{He}$ & $\mathrm{GF}, \mathrm{FB}$ & $12-5$ & $4-11$ \\
\hline Coccocypselum lanceolatum (Ruiz and Pav.) Pers. & 16374 & $\mathrm{He}$ & GF & $12-5$ & $3-6$ \\
\hline Ferdinandusa speciosa Pohl & 16385 & $\mathrm{Sh}$ & FB & $4-5,9$ & $4-5$ \\
\hline Galium noxium (A. St.-Hil.) Dempster & 19387 & $\mathrm{He}$ & FB & $1-2$ & - \\
\hline Palicourea crocea (Sw.) Roem. and Schult. & 17711 & Ss & GF & $7-5$ & $1-7$ \\
\hline Psychotria capitata Ruiz and Pav. & 19255 & $\mathrm{Sh}$ & GF & $10-7$ & $12-8$ \\
\hline Psychotria hoffmannseggiana (Willd. ex Schult.) Müll. Arg. & 16376 & Ss & GF & $9-5$ & $12-6$ \\
\hline Psychotria subtriflora Müll. Arg. & 19241 & Ss & GF & $5-3$ & $10-5$ \\
\hline Psychotria vellosiana Benth. & 19305 & Ss & GF & $11-12$ & 12 \\
\hline \multicolumn{6}{|l|}{ Siparunaceae } \\
\hline Siparuna brasiliensis (Spreng.) A. DC. & 19344 & Sh & GF, FB & 12 & $12-1$ \\
\hline \multicolumn{6}{|l|}{ Solanaceae } \\
\hline Brunfelsia brasiliensis (Spreng.) L. B. Sm. and Downs & 19368 & Sh & GF & $1-2$ & - \\
\hline \multicolumn{6}{|l|}{ Turneraceae } \\
\hline Piriqueta sidifolia (Cambess.) Urb. & 19356 & Ss & $\mathrm{FB} *$ & 1,5 & 5 \\
\hline Turnera oblongifolia Cambess. & 19464 & Ss & $\mathrm{FB}$ & 5 & - \\
\hline \multicolumn{6}{|l|}{ Velloziaceae } \\
\hline Vellozia squamata Pohl & 16388 & $\mathrm{Sh}$ & $\mathrm{FB} *$ & $4-5$ & $5-9$ \\
\hline
\end{tabular}




\begin{tabular}{|c|c|c|c|c|c|}
\hline FAMILY/SPECIES & UB & $\mathbf{L F}$ & Habitat & Fl. & Fr. \\
\hline $\begin{array}{l}\text { Verbenaceae } \\
\text { Lippia rotundifolia } \text { Cham. }\end{array}$ & 17725 & Ss & FB* & $9-1$ & 1 \\
\hline $\begin{array}{l}\text { Xyridaceae } \\
\text { Xyris hymenachne Mart. } \\
\text { Xyris jupicai } \text { Rich }\end{array}$ & $\begin{array}{l}19364 \\
19375\end{array}$ & $\begin{array}{l}\mathrm{He} \\
\mathrm{He}\end{array}$ & $\begin{array}{l}\text { FB* } \\
\text { GF }\end{array}$ & $\begin{array}{l}1 \\
2\end{array}$ & - \\
\hline
\end{tabular}

* species collected along border of the Gallery Forest, however also typical in other phytophysionomies such as: campo limpo úmido, vereda or cerrado sensu stricto.

** Species not cited by Munhoz and Proença (1998) and Mendonça et al (2008).

Similar results were found by Mendonça et al. (2000), in a preliminary survey of the flora in the core areas of the Cerrado Reserve, Federal District, including the IBGE Ecological Reserve and National Park of Brasilia, showing that for these habitats, $36.4 \%$ of species in gallery forests were found in these same five families. It should be noted that Mendonça et al. (2000) included epiphytes within the herbaceous habit, which were not included in this work, leading to some distortion in the compared data. This could be very significant, since over half the species of the orchid family (one of the largest) in gallery forests, were epiphytes (Felfili et al. 2001). Therefore, without these differences in the methodology, the percentages of the five families would be even closer when comparing the two studies. Asteraceae, Poaceae, Rubiaceae, Melastomataceae and Fabaceae, have also been cited among the most species-rich families both for the Cerrado biome (Mendonça et al. 2000; Mendonça et al. 2008; Munhoz and Proença 1998), and also for the riverine and gallery forests (Felfili et al. 2001), which indicated their wide representation and distribution in the context of the biome.

The families with highest number of genera were Asteraceae (19 genera), Fabaceae (8), Poaceae (8) and Rubiaceae (7), totaling $40.8 \%$ of all genera sampled. Among the genera, the most representative were Ichnanthus (Poaceae), Piper (Piperaceae) and Psychotria (Rubiaceae), with four species each. The species distribution in the studied area was: 46 species $(33.3 \%)$ restricted to inside the forest; $82(59.4 \%)$ only in the forest border; and 10 species were registered on both the sites. Among the border species, 53 species (38.4\%) were typical of open areas, such as campo limpo úmido, vereda and Cerrado sensu stricto (Munhoz and Proença 1998; Mendonça et al. 2008). Twenty nine (21\%) species were typical of gallery forest border.
Despite a very rich flora, only two species, Chresta angustifolia and Cybianthus goyazensis were endemics to the Goiás State. It is notable that 52 of the species found were not cited for the Gallery forest or its border by Silva et al. (1996), Munhoz and Proença (1998), Mendonça et al. (2000), Felfili et al. (2001), Proença et al. (2001) and Mendonça et al. (2008) studies. These authors recorded no representatives of the Xyridaceae family in the gallery forests, while in this study, samples of Xyris jupicai were collected on the banks of a small stream, in a tributary of the Couros river and Xyris hymenachne along the gallery forest border. Felfili et al. (2001), Mendonça et al. (2000) and Silva et al. (1996) reported that, in general, the herbaceous shrub layer has a higher percentage of woody or semiwoody plants than herbs in riparian and gallery forests. In the present study, similar results were found, with $43.5 \%$ of the plants sampled in this layer being herbaceous.

Over the 25 sampling days, some species were frequently found, while others were recorded only once. Coccocypselum lanceolatum, Justicia irwinii, Palicourea crocea, Piper xylosteoides, Psychotria capitata and Scleria latifolia were found relatively easily at various points in the forest. The species Alstroemeria stenopetala, Bromelia antiacantha, Coccocypselum aureum and Miconia albicans were also found easily, however, in small isolated areas. Epidendrum secundum, Leandra salicina, Oxalis diamantinae, Pavonia malacophylla, Psychotria vellosiana and Sinningia elatior were rare in the Couros river gallery forest.

The data obtained in this study were compared with those in Silva et al. (2001) and Munhoz and Proença (1998), who studied the areas near to the Portal da Chapada in the Chapada dos Veadeiros and showed a significant increase in the number of species in the region. It was observed that 65 
species $(47 \%)$ had not yet been reported for the flora of the region based on these authors. According to the same studies, 123 species were not included as part of the gallery forests neither in the interior or along the border.

Out of the total number of species cited by Munhoz and Proença (1998) for the Chapada dos Veadeiros region, 57 were found in the herbaceous-shrub layer of Gallery Forest and its border, and 14 of them found in this study. Therefore, the increases in the flora were $5 \%$ for the region as a whole, $99.2 \%$ for the gallery forests and $223.6 \%$ for the herbaceous-shrub layer of the gallery forests in the Chapada dos Veadeiros region. The data provided here showed the lack of studies in the region, particularly for life forms. The richness observed in this study could be related to the systematization of the study, allowing the sampling of the species throughout the year in different phenological phases.

\section{ACKNOWLEDGEMENTS}

The authors would like to thank Professor Dr. Carolyn Proença up to 2009, the curator of the UB Herbarium, Mr. Antonio Cabral, owner of the Portal da Chapada for logistical support; Mr. Wandélio Mendes and collaborators for their support during field work; and to the botanical specialists for confirmation of the taxa.

\section{REFERENCES}

APG III. An update of the Angiosperm Phylogeny Group classification for the orders and families of flowering plants: APG III. Bot J Linnean Soc. 2009; 161: $105-21$.

Assis MC. Alstroemeriaceae. In: Cavalcanti TB \& Ramos AE, editors. Flora do Distrito Federal, Brasil. v2. Brasília: EMBRAPA-CENARGEN; 2002. p. 2132.

Barbosa AVG, Amaral Júnior. A. Flora do Estado de Goiás e Tocantins: Erythroxylaceae. Coleção Rizzo 29. UFG; 2001.

Barroso GM, Guimarães EF, Ichaso CLF, Costa CG, Peixoto AL. Sistemática de Angiospermas do Brasil. v1. Viçosa: Editora da UFV; 2002.

Carvalho-Silva M \& Cavalcanti TB. Piperaceae. In: Cavalcanti TB \& Ramos AE. editors. Flora do Distrito Federal, Brasil. v2. Brasília: EMBRAPACENARGEN; 2002. p. 93-124.
Cavalcanti RB. Coordenação Cerrado e Pantanal. In: Biodiversidade Brasileira. Brasília: MMA-SBF; 2002.

Cavalcanti TB, Graham SA, Silva MC. Flora do Estado de Goiás e Tocantins: Lythraceae. Coleção Rizzo 28. UFG; 2001.

Chautems A. Gesneriaceae. In: Cavalcanti TB \& Ramos AE. editors. Flora do Distrito Federal, Brasil. v3. Brasília: EMBRAPA-CENARGEN; 2003. p. 185-97.

Correia JR, Haridasan M, Reatto A, Martins ES, Walter BMT. Influência de fatores edáficos na distribuição de espécies arbóreas em Matas de Galeria na região do Cerrado: uma revisão. In: Ribeiro JF, Fonseca CEL, Sousa-Silva JC. editors. Cerrado: caracterização e recuperação de Matas de Galeria. Planaltina: EMBRAPA-CERRADOS; 2001. p. 51-76.

Eira MTS, Martins Netto DA. Germinação e conservação de sementes de espécies lenhosas. In: Ribeiro JF. editor. Cerrado: Matas de Galeria. Planaltina: EMBRAPA-CPAC; 1998. p. 95-117.

Eiten G. Formas de crescimento das plantas vasculares. In: HERBÁRIO UB, $2^{\circ}$ Boletim Informativo. Brasília: UnB; 1992. p. 6-13.

Felfili JM, Mendonça RC, Walter BMT, Silva Júnior $\mathrm{MC}$, Nóbrega MGG, Fagg $\mathrm{CW}$, et al. Flora fanerogâmica das Matas de Galeria e Ciliares do Brasil Central. In: Ribeiro JF, Fonseca CEL, SousaSilva JC. editors. Cerrado: caracterização e recuperação de Matas de Galeria. Planaltina: EMBRAPA-CERRADOS; 2001. p. 195-263.

Filgueiras TS. Flora do Estado de Goiás e Tocantins: Gramineae (Poaceae). Coleção Rizzo 17. UFG; 1995.

Fonseca CEL, Ribeiro JF. Produção de mudas e crescimento inicial de espécies arbóreas. In: Ribeiro JF editor. Cerrado: Matas de Galeria. Planaltina: EMBRAPA-CPAC; 1998. p. 119-33.

Haridasan M. Solos de matas de galeria e nutrição mineral de espécies arbóreas em condições naturais. In: Ribeiro JF. editor. Cerrado: Matas de Galeria. Planaltina: EMBRAPA-CPAC; 1998. p. 17-28.

Judd WS, Campbell CS, Kellogg EA, Stevens PF. Plant Systematics: a phylogenetic approach. Sunderland: Sinauer Associates, Inc.; 1999.

Lombardi JA. Vitaceae. In: Cavalcanti TB \& Ramos AE. editors. Flora do Distrito Federal, Brasil. v2. Brasília: EMBRAPA-CENARGEN; 2002. p. 143-53.

Mendonça JO, Amaral Júnior A. Erythroxylaceae. In: Cavalcanti TB, Ramos AE. editors. Flora do Distrito Federal, Brasil. v2. Brasília: EMBRAPACENARGEN; 2002. p. 53-71.

Mendonça RC, Felfili JM, Silva JCS. Diversidade e composição florística das áreas nucleares da Reserva da Biosfera do Cerrado - Fase I. In: Schenkel CS, Brummer BM. editors. Vegetação no Distrito Federal: tempo e espaço. Brasília: UNESCO; 2000. p. 31-2. 
Mendonça RC, Felfili JM, Walter BMT, Silva Júnior MC, Rezende AV, Filgueiras TS, et al. Flora vascular do Cerrado. In: Sano SM, Almeida SP. editors. Cerrado: Ambiente e flora. Planaltina: EMBRAPACPAC; 1998. p. 290-556.

Munhoz CBR, Proença CEB. Composição florística do município de Alto Paraíso de Goiás na Chapada dos Veadeiros. Bol Herb Ezechias Paulo Heringer. 1998; 3: $102-50$.

Nogueira PE, Nóbrega MGG, Silva GP. Levantamento florístico e fisionomias do Parque Ecológico Ezechias Heringer (Parque do Guará), Distrito Federal, Brasil. Bol Herb Ezechias Paulo Heringer. 2002; 10:31-56.

Proença CEB, Munhoz CBR, Jorge CL, Nóbrega MGG. Listagem e nível de proteção das espécies de fanerógamas do Distrito Federal, Brasil. In: Cavalcanti TB, Ramos AE. editors. Flora do Distrito Federal, Brasil. v1. Brasília: EMBRAPACENARGEN; 2001. p. 89-359.

Rezende AV. Importância das matas de galeria: manutenção e recuperação. In: Ribeiro JF. editor. Cerrado: Matas de Galeria. Planaltina: EMBRAPACPAC; 1998. p. 95-117.

Ribeiro JF, Schiavini I. Recuperação das Matas de Galeria: integração entre a oferta ambiental e a biologia das espécies. In: Ribeiro JF. editor. Cerrado: Matas de Galeria. Planaltina: EMBRAPA-CPAC; 1998. p. 135-53.

Ribeiro JF, Walter BMT. Fitofisionomias do bioma Cerrado. In: Sano SM, Almeida SP. Editor. Cerrado: Ambiente e flora. Planaltina: EMBRAPA-CPAC; 1998. p. 89-166.

Ribeiro JF, Walter BMT. As Matas de Galeria no contexto do Bioma Cerrado. In: Ribeiro JF, Fonseca CEL, Sousa-Silva JC. editors. Cerrado: caracterização e recuperação de Matas de Galeria. Planaltina: EMBRAPA-CERRADOS; 2001. p. 29-47.

Schiavini I, França JC, Aquino FG. Dinâmica de populações de espécies arbóreas em mata de galeria e mata mesófila na margem do Ribeirão Panga, MG. In: Ribeiro JF, Fonseca CEL, Sousa-Silva JC. editors. Cerrado: caracterização e recuperação de Matas de Galeria. Planaltina: EMBRAPA-CERRADOS; 2001. p. 265-299.
Silva BA, Mecenas VV, Leite FQ, Cardoso ES. Apa de Cafuringa: o retrato do cerrado. Brasília: Paralelo 15; 1996.

Silva SR, Silva AP, Munhoz CB, Silva Júnior MC, Medeiros MB. Guia de plantas do cerrado utilizadas na Chapada dos Veadeiros. Brasília: WWF; 2001.

Silva Júnior MC, Felfili JM, Nogueira PE, Rezende AV. Análise florística das Matas de Galeria no Distrito Federal. In: Ribeiro JF editor. Cerrado: Matas de Galeria. Planaltina: EMBRAPA-CPAC; 1998. p. 51-84.

Silva Júnior MC, Felfili JM, Walter BMT, Nogueira PE, Rezende AV, Morais RO et al. Análise da flora arbórea de Matas de Galeria no Distrito Federal: 21 levantamentos. In: Ribeiro JF, Fonseca CEL, SousaSilva JC. editors. Cerrado: caracterização e recuperação de Matas de Galeria. Planaltina: EMBRAPA-CERRADOS; 2001. p. 143-91.

Soares-Silva LH. Levantamento florístico das espécies arbóreas do Portal da Chapada, Alto Paraíso, GO. In: Congresso Nacional de Botânica; 2005; Curitiba. Anais do Congresso Nacional de Botânica; 2005.

Souza CD. Florística, fitossociologia e etnobotânica na região do entorno do Parque Nacional da Chapada dos Veadeiros, Goiás, Brasil. [Dissertação de Mestrado]. Brasil: Universidade de Brasília; 1999.

Souza VC, Lorenzi H. Botânica Sistemática. Nova Odessa: Instituto Plantarum de Estudos da Flora LTDA; 2005.

Wanderley, M. das G. L. 1989. Flora do Estado de Goiás e Tocantins: Xyridaceae. Coleção Rizzo 11. UFG. 